\title{
Volatile Blood Pressure Due To Baroreflex and Autonomic Failure
}

\author{
Mauro Vasella, Verena Briner \\ Department of Medicine, Luzerner Kantonsspital, Lucerne, Switzerland
}

\begin{abstract}
An 85-year-old male was hospitalized because of deterioration of his general condition and infection of the tracheostoma. He had had laryngectomy, bilateral neck dissection and radiation therapy for a laryngeal carcinoma 5 years earlier. Despite a good recovery, he could not get up because of a new onset of postural symptoms (dizziness, lightheadedness, collapse). Late onset of baroreflex failure and autonomic nervous system failure were diagnosed. Volatility of blood pressure (supine hypertension, upright hypotension) was treated with $\mathrm{NaCl}$ supplement during the day and a short-acting antihypertensive (clonidine) at night. With this regimen, the patient could walk without support.
\end{abstract}

Keywords: Baroreflex and autonomic failure, volatile blood pressure

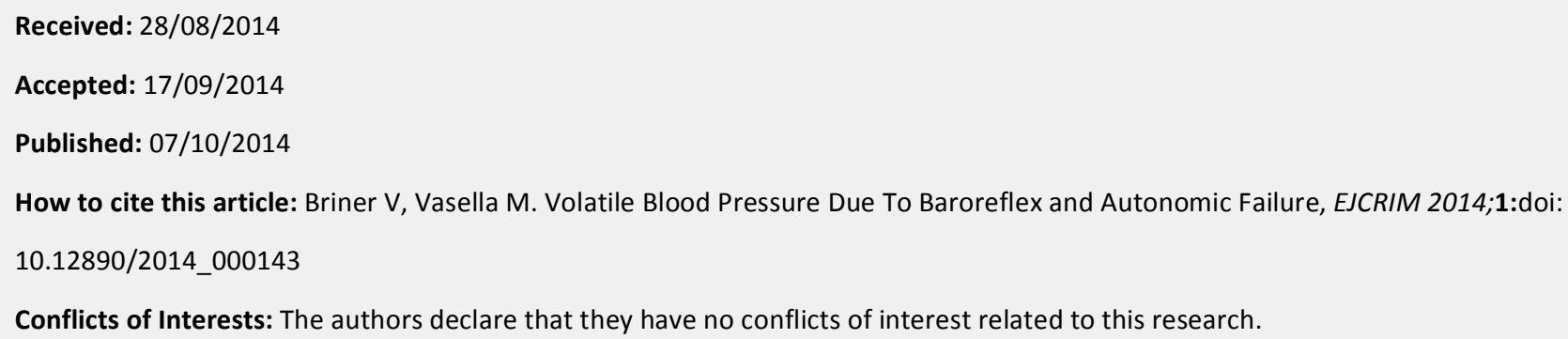

Conflicts of Interests: The authors declare that they have no conflicts of interest related to this research.

\section{Introduction}

Orthostatic collapse is very frequent. The aetiology may differ, e.g. hypovolaemia, drugs that limit vasoconstriction, autonomic nervous system (ANS) failure. ANS is a complex disorder and may be the result of neurodegenerative diseases or neuropathies [1]. A less common aetiology of a collapse results from baroreflex injury [2]. Baroreceptors in the carotid sinus and great vessels of the thorax are mechanoreceptors that are important to rapidly buffer fluctuation of blood pressure to avoid hyper- and hypotension. Afferent fibres of the glossopharyngeal and vagal nerve, respectively, respond to blood pressure changes and connect to the medulla in the brain stem. Sympathetic and parasympathetic fibres serve as efferent pathways to regulate the homeostasis of blood pressure and heart rate. Depending on the site and severity of any damage, impaired regulation of blood pressure and heart rate, but also gastroparesis, obstipation and urine retention may occur [3]. We describe a patient with sudden onset of disabling orthostatic hypotension. 


\section{Case report}

An 85-year-old Caucasian male was hospitalized because of worsened general condition and tracheostomy with purulent discharge. Five years earlier, he had had a laryngectomy, bilateral neck dissection and radiation therapy for a laryngeal cancer. There were no signs of tumour recurrence (CT scan, laryngoscopy). The tracheal cannula was changed and antibiotics were given. The infection resolved; however, the patient felt dizzy when trying to get up and collapsed even when wearing compression stockings. There were no signs of volume depletion, a cardiac cause (troponin T, echocardiography) or an epilepsy (electroencephalography). The CT angiography showed normal vertebral and basilar arteries. A 24-h electrocardiography was recorded, when the patient fainted, documenting a regular sinus rhythm but a missing variability of the circadian heart rate. Immediately after fainting, the blood pressure was $90 / 70 \mathrm{mmHg}$, with a stable pulse rate $(60 \mathrm{bpm})$. Saline infusion was given. Even though the blood pressure rose and the patient's condition improved, he collapsed again when saline was stopped and therefore it was continued. Hypertension developed when the patient was supine $(>220 / 110 \mathrm{mmHg}$ ). The drug treatment was adapted, but no combination was able to stabilize the blood pressure. A pheochromocytoma (blood metanephrine and normetanephrine) and a mastocytosis (blood tryptase) were excluded.

Figure 1 demonstrates that within less than $5 \mathrm{~s}$ after getting up, blood pressure dropped from 139/63 to 98/66 $\mathrm{mmHg}$ and the patient fainted. Before collapsing, he had dizziness and blurred vision. When he went back to a supine position,

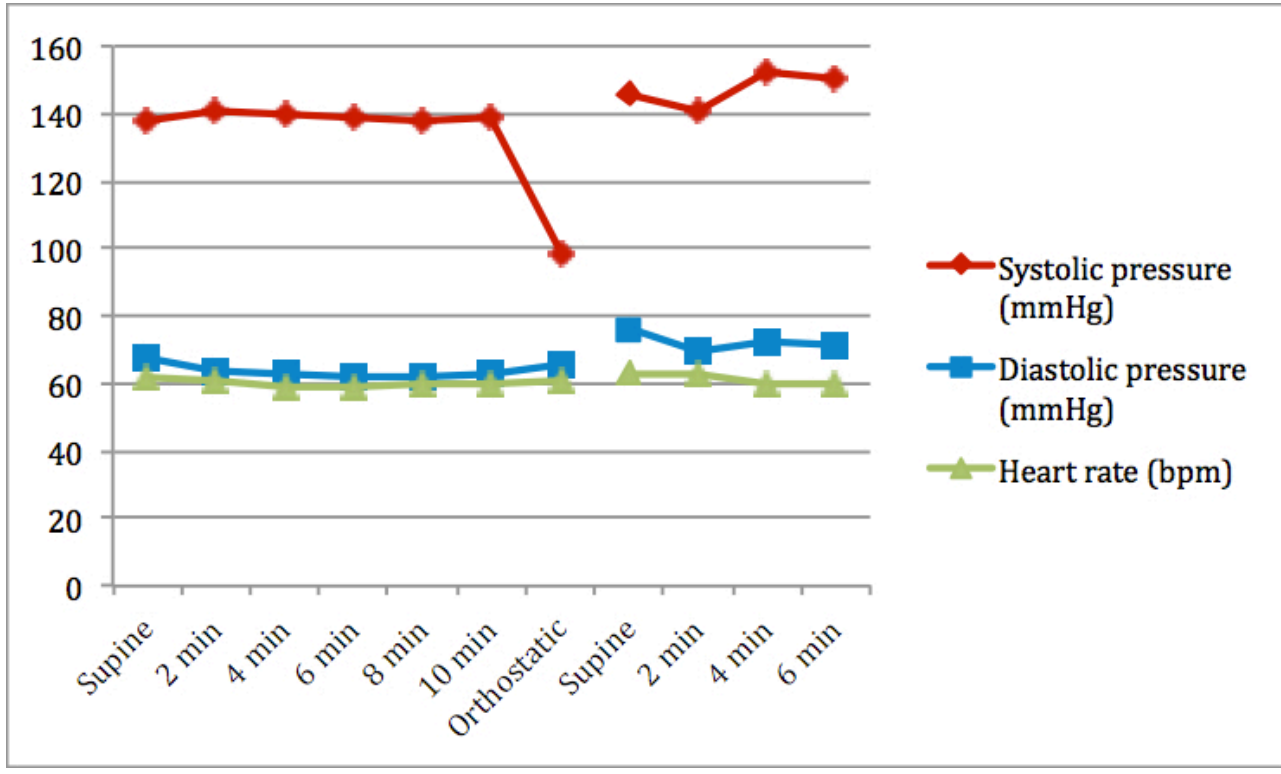

Figure 1: Changes of systolic and diastolic blood pressure and heart rate while changing postural positions from supine to upright. the blood pressure was 152/72 $\mathrm{mmHg}$ and the heart rate $60 \mathrm{bpm}$. The symptoms disappeared immediately. The Valsalva manoeuvre did not show a significant change in the blood pressure (119/69 to $115 / 72 \mathrm{mmHg})$ or the heart rate (61 to $59 \mathrm{bpm}$ ), indicating an impaired baroreflex. The patient also had dysphagia, disturbed oesophageal motility (video analysis), constipation and urinary retention, suggesting an ANS dysfunction. There were no signs of diabetes, Parkinson's

disease, amyloidosis or vitamin B12 deficiency. A paraneoplastic aetiology was therefore the most likely cause of the syndrome. The patient was treated with $1 \mathrm{~g} \mathrm{NaCl}$ tablet in the morning and $0.5 \mathrm{~g}$ at noon to increase intravascular volume during the daytime. He also wore compression stockings. In the evening, a short-acting antihypertensive drug was initiated (clonidine $75 \mu \mathrm{g}$ ) and the headrest was elevated to $30^{\circ}$. Blood pressure fluctuation was reduced: supine 145/65 mm $\mathrm{mg}$, sitting 142/66 mmHg and standing 135/66 mmHg, without a change in the heart rate (58, 59 and $59 \mathrm{bpm}$, respectively). He was able to walk without support. 


\section{Discussion}

Postural change from supine to standing may cause a pooling of $>300 \mathrm{ml}$ of blood in the legs, resulting in a low cardiac output and a drop of blood pressure with subsequent baroreceptor activation and signal transmission to the brain stem. Sympathetic efferent outflow to the heart and blood vessels lead to an appropriate rise in heart rate and vasoconstriction to maintain blood pressure and cardiac output. It has been shown that tumour growth, radiotherapy, trauma or surgery of the neck damaging the carotid sinus or glossopharyngeal nerve, and stroke of the brainstem all can cause baroreflex failure. However, the clinical presentation depends on the degree and site of the damage. Acute baroreflex failure after bilateral carotid sinus removal causing a glossopharyngeal loss leads to severe hypertension due to damage to the efferent parasympathetic output to tachycardia. When a baroreflex failure occurs without damage and the parasympathetic fibres of the vagal nerve, severe bradycardia, hypotension and even sinus arrest may result. The onset of symptoms of baroreflex failure can occur even years after treatment of laryngeal cancer. Local fibrosis, arterial stiffness [4] and atherosclerosis of the carotid sinus area may be involved.

Autonomic failure impairs the sympathetic, parasympathetic and enteric nervous systems in various combinations. Motility of the intestinal tube depends on reflexes of the enteric nervous system and is modulated by the vagus nerve and sympathetic inputs. When impaired, delayed oesophageal transit, gastric retention and constipation may result. These signs have been observed in our patient, most likely due to paraneoplastic aetiology. The vagal control over the sinus node is assessed by the change of the heart rate while performing deep breaths or a Valsalva manoeuvre. Reflex tachycardia may result from a loss of vagal parasympathetic input to the sinus node. A regular Valsalva manoeuvre causes an initial increase in systolic blood pressure and a decrease in heart rate (phase 1), followed by a return to baseline combined with little increase in heart rate (phase 2). When pressure is released, blood pressure drops below baseline (phase 3) and is followed by an overshooting rise of blood pressure and bradycardia (phase 4) [5]. Phase 4 is the result of a normal baroreflex interaction. This was missing in our patient, thus supporting the diagnosis of baroreflex failure and the respiration-induced sinus arrhythmia, suggesting that the parasympathetic path to the heart was impaired. The function of the sympathetic nervous system can be analyzed by the beat-to-beat blood pressure changes (puls rate) during the Valsalva manoeuvre. This variability was lost in our patient suggesting sympathetic nervous system failure too.

\section{Conclusion}

The diagnosis of failure of baroreflex and autonomic nervous system might easily be missed because symptoms are non-specific and may start some time after treatment of laryngeal carcinoma. It is crucial to monitor blood pressure and heart rate in supine, sitting and standing positions to prevent inadequate drug treatment exaggerating the imbalance and thus worsening symptoms. To reduce supine hypertension, a short-acting antihypertensive, e.g. clonidine $\left(\alpha_{2}\right.$-adrenoreceptor agonist) or captopril (ACEI), are recommended, while diuretics, $\alpha$-adrenoreceptor blockers and vasodilators should be avoided. Heightening the head to about $30^{\circ}$ while in supine position prevents volume mobilization from the legs during night-time. The treatment goal is to reduce the risk of falls and stop damage to organs. 


\section{European Journal}

of Case Reports in

Internal Medicine

\section{Learning Points}

- The response to a change in posture depends on the baroreflex and the autonomic nervous system. Damage to one or more sites may impair blood pressure regulation.

- The history and bedside tests may lead to the diagnosis of an impaired baroreflex and autonomic failure.

- Supine hypertension and orthostatic hypotension are treated with a combination of volume expansion during the daytime and pressure-lowering drugs at night.

\section{References}

1. Benarroch EE. The clinical approach to autonomic failure in neurological disorders, Nat Rev Neurol 2014;10:396407.

2. Robertson D, Hollister AS, Biaggioni I, Netterville JI, Mosqueda-Garcia R, Robertson RM. The diagnosis and treatment of baroreflex failure, New Engl J Med 1993;329:1449-1455.

3. Ketch T, Biaggioni I, Robertson RM, Robertson D. Four faces of Baroreflex failure: hypertensive crisis, volatile hypertension, orthostatic tachycardia and malignant vagotonia, Circulation 2002;105:2518-2523.

4. Michas F, Manios E, Stamatelopoulos K, Koroboki E, Toumanidis S, Panerai RB et al. Baroreceptor reflex sensitivity is associated with arterial stiffness in a population of normotensive and hypertensive patients, Clin Meth Pathophysiol 2012;17:155-159.

5. Biaggioni I. Hypertension in orthostatic hypotension and autonomic dysfunction, Cardiol Clin 2002;20:291-301. 